\title{
Intracranial Arteriovenous Shunting Detection with Arterial Spin-Labeling and Susceptibility-Weighted Imaging: Potential Pitfall of a Venous Predominant Parenchymal Arteriovenous Malformation
}

l read with great interest the study of Hodel et al, ${ }^{1}$ which demonstrated that the combined use of arterial spin-labeling (ASL) and susceptibility-weighted imaging was significantly more sensitive and equally specific compared with conventional angiographic MR imaging for the detection of arteriovenous shunting. In Fig 2 of this article, a lesion with increased signal intensity on ASL images was subsequently confirmed as a developmental venous anomaly (DVA) based on the presence of a classic umbrellashaped appearance on the venous phase cerebral angiogram. ${ }^{1}$

Most DVAs are not associated with perfusion changes on ASL imaging. In a large study of 652 DVAs, only a minority of DVAs (8\%) demonstrated signal abnormalities on ASL maps, ${ }^{2}$ and intrinsically increased ASL signal or increased signal in a draining vein associated with a DVA are potentially imaging biomarkers of arteriovenous shunting. ${ }^{2,3}$ It may be argued that the presence of a typical venous phase cerebral angiogram with a caput medusa appearance will confirm the DVA. However, direct arteriovenous shunting into dilated medullary veins characteristic of a DVA without a typical nidus has been repeatedly reported in the literature. These arterialized DVAs have been described with various terminology, but Im et $\mathrm{al}^{4}$ proposed the name of "venous-predominant parenchymal AVMs" on the basis of clinical, angiographic, surgical, and histologic findings in a series of 15 cases. Of note, the cerebral angiograms of these lesions demonstrated an abnormal blush in the early arterial phase, followed by diffuse vascular and capillary parenchymal staining in the mid-arterial phase and immediate drainage into a network of radially arranged

http://dx.doi.org/10.3174/ajnr.A5108 dilated medullary veins. ${ }^{4}$ These lesions consistently showed a caput medusa appearance very similar to the appearance of DVAs, with the absence of enlarged arterial feeders and lack of a typical AVM nidus.

In summary, a lesion with increased signal intensity on an ASL map is suspicious for arteriovenous shunting, and a caput medusa appearance on the venous phase of a cerebral angiogram is not sufficient to make the diagnosis of DVA. Careful attention to the arterial phase of the angiogram would be prudent to exclude a venous-predominant parenchymal arteriovenous malformation.

\section{REFERENCES}

1. Hodel J, Leclerc X, Kalsoum E, et al. Intracranial arteriovenous shunting: detection with arterial spin-labeling and susceptibilityweighted imaging combined. AJNR Am J Neuroradiol 2017;38:71-76 CrossRef Medline

2. Iv M, Fischbein NJ, Zaharchuk G. Association of developmental venous anomalies with perfusion abnormalities on arterial spin labeling and bolus perfusion-weighted imaging. J Neuroimaging 2015;25: 243-50 CrossRef Medline

3. Nabavizadeh SA, Mamourian AC, Vossough A, et al. The many faces of cerebral developmental venous anomaly and its mimicks: spectrum of imaging findings. J Neuroimaging 2016;26:463-72 CrossRef Medline

4. Im SH, Han MH, Kwon BJ, et al. Venous-predominant parenchymal arteriovenous malformation: a rare subtype with venous drainage pattern mimicking developmental venous anomaly. J Neurosurg 2008;108:1142-47 CrossRef Medline

(D.A. Nabavizadeh Department of Radiology Hospital of University of Pennsylvania Perelman School of Medicine at the University of Pennsylvania Philadelphia, Pennsylvania 